\title{
Quality of Life and Mortality Assessment in Patients with Major Cardiac Events in the Postoperative Period
}

\author{
Fernando José Abelha ${ }^{1}$, Miguela Botelho ${ }^{2}$, Vera Fernandes ${ }^{2}$, Henrique Barros ${ }^{3}$
}

Summary: Abelha FJ, Botelho M, Fernandes V, Barros H - Quality of Life and Mortality Assessment in Patients with Major Cardiac Events in the Postoperative Period.

Background and objectives: Cardiovascular complications in the postoperative period are associated with high mortality and morbidity. Few studies have assessed the degree of dependence in these patients and their perception of health. The objective of this study was to assess the mortality and the quality of life in patients who developed major cardiac events (MCE) in the postoperative period.

\begin{abstract}
Method: Retrospective study carried out in a Surgical Intensive Care Unit (SICU), between March 2006 and March 2008. The patients were assessed regarding the occurrence of CE. Six months after the hospital discharge, the Short-Form-36 (SF-36) questionnaire was filled out and dependence was assessed in relation to activities of daily living (ADL). The comparisons between independent groups of patients were carried out using Student's t test. The comparison between each variable and the occurrence of CE was carried out by logistic regression and included all patients.

Results: Of the 1,280 patients that met the inclusion criteria, 26 (2\%) developed MCE. The univariate analysis identified as independent determinants for the development of major cardiac events: ASA physical status, hypertension, ischemic heart disease, congestive heart disease and score of the Revised Cardiac Risk Index (RCRI). The six-month mortality after the SICU discharge was 35\%. Of the 17 surviving patients, 13 completed the questionnaires. Thirty-one percent of them reported that their general health was better on the day they answered the questionnaire, when compared to 12 months before. Sixty-nine percent of the patients were dependent in instrumental ADL e 15\% in personal ADL.
\end{abstract}

Conclusions: The development of MCE has a significant impact on the duration of hospital stay and mortality rates. Six months after the discharge from the SICU, more than $50 \%$ of the patients were dependent in at least one instrumental ADL.

Keywords: COMPLICATIONS: morbidity, mortality, postoperative; SURGERY, Cardiac: quality of life.

[Rev Bras Anestesiol 2010;60(3): 268-284] CElsevier Editora Ltda.

\section{INTRODUCTION}

The high prevalence of cardiac disease in the population has major implications in the postoperative setting, and the risk of adverse cardiac events can be significant for patients undergoing major surgery. For instance, cardiac death, myocardial infarction, myocardial ischemia, congestive heart failure and arrhythmias are associated with increased mortality and morbidity and result in longer hospital stay and substantially higher treatment costs ${ }^{1}$. In the early postoperative period, cardiac events may occur without symptoms or distinctive electrocardiographic changes and may go unrecognized, so accurate assessment of perioperative cardiac risk can guide

\footnotetext{
Received from Serviço de Anestesiologia Unidade Pós-Anestésica do Hospital de São João, Porto, Portugal

1. Anesthesiology and Intensive Care - Chief of the Service of Anesthesiology

2. Anesthesiologist

3. Department of Hygiene and Epidemiology, Faculdade de Medicina do Porto - Director of the service; PhD, Professor

Submitted on October 7, 2009

Approved on February 8, 2010

Correspondence to:

Dr. Fernando José Abelha

Serviço de Anestesiologia

Hospital de São João

Alameda Professor Hernani Monteiro, Porto, Portugal

E-mail: fernando.abelha@gmail.com
}

prophylactic treatment, perioperative management, location and intensity of postoperative care and early detection of cardiac complications.

Some perioperative studies have focused on patients with or at risk of cardiac disease ${ }^{2,3}$, but few on unselected patients subjected to all types of non-cardiac surgery ${ }^{4}$. Lee et al. ${ }^{5}$ evaluated 4,315 patients aged 50 years with expected postoperative length of stay equal or higher than 2 days, but excluded patients undergoing emergency surgery.

Few studies have examined the dependency of these patients and their perceptions of their own health. Several questionnaires have been validated for the study of Health Related Quality of Life (HRQOL) ${ }^{6-10}$. Most of the measures used are multi-item scales; that is, they are made up of several questions or items. Some multiple-item scales provide a total score as well as generating subscales that provide information on particular aspects such as mobility. The Short-Form General Health Survey (SF-36) was developed during the Medical Outcomes Study (MOS) to measure generic health concepts relevant across age, disease and treatment groups ${ }^{11}$. It is a valid instrument for measuring HRQOL that has been used for post-discharge ICU patients and in the study of groups with other diseases; it shows good reliability and validity ${ }^{11,12}$. This questionnaire has been culturally adapted to Portuguese and validated in a study by Ferreira ${ }^{13,14}$. 
Low functional status puts patients at higher risk. Patients with no or only minor clinical predictors but with poor functional capacity are recommended to undergo noninvasive testing prior to surgery. The ability to care for oneself and live independently is considered a measure of functional outcome after hospitalization ${ }^{15}$. Functional status refers to the level of involvement in activities and is often used as a synonym for performance in Activities of Daily Living (ADL) ${ }^{16}$. ADL appraisal scales consider functional and instrumental activities. The ability of patients to handle these activities has been assessed by generic or disease-specific tests. Katz's ADL Scale ${ }^{16}$ and the Lawton Instrumental ADL ${ }^{17}$ have been used for critical care survivors.

The aim of the present study was to evaluate outcome, quality of life and autonomy in ADL in patients who develop major cardiac events (MCE) after major surgery.

\section{METHODS}

This retrospective cohort study was conducted at a multidisciplinary Post-Anesthesia Care Unit (PACU). Included in the PACU is a Surgical Intensive Care Unit (SICU) with five beds, in which critically ill surgical patients are admitted and closely monitored and treated.

All postoperative patients admitted to the SICU area of the PACU, aged 18 years or over, who underwent scheduled or emergency surgery between 1 March 2006 and 1 March 2008 with an overnight admission and more than $12 \mathrm{~h}$ of PACU stay were eligible for inclusion in the study. Patients readmitted during the study period were enrolled in relation to the time of their first admission. Patients subjected to cardiac or pulmonary surgery were excluded.

The following variables were recorded on admission to the PACU: age, gender, body mass index (BMI), American Society of Anesthesiologists physical status (ASA-PS) ${ }^{18}$, emergency or scheduled surgery, preadmission comorbidities (specifically ischemic heart disease, congestive heart failure, cerebrovascular disease, hypertension, renal insufficiency, diabetes, hyperlipidemia), duration and type of anesthesia, core temperature and blood troponin I levels. Intraoperative data recorded for each case included administration of crystalloids, colloids, erythrocytes and fresh frozen plasma.

Postoperative cardiac troponin I (cTnl) and the occurrence of major cardiac events (MCE) were recorded. Intraoperative and PACU data were collected as well as hospital length of stay (LOS). Mortality was recorded for all patients. The Acute Physiology and Chronic Health Evaluation (APACHE) II ${ }^{19}$ and the Simplified Acute Physiology Score II (SAPS II) ${ }^{20}$ were calculated using standard methods. Six months after discharge, patients were contacted to complete a Short Form-36 questionnaire (SF-36) and to have their dependency in activities of daily living (ADL) evaluated.

Using a classification scheme developed by Lee and colleagues to predict cardiac risk, we calculated a Revised Cardiac Risk Index (RCRI) score for each patient, assigning one point for each of the following risk factors: high risk surgery (thora- cic, abdominal, or pelvic vascular e.g. aorta, renal, mesenteric), history of ischemic heart disease, history of congestive heart failure, history of cerebrovascular disease, preoperative treatment with insulin, and preoperative serum creatinine > $2.0 \mathrm{mg} \cdot \mathrm{dL}^{-1} 5$.

Acute myocardial infarction, pulmonary edema, ventricular fibrillation or primary cardiac arrest and complete heart block were recorded during PACU stay and considered to be Major Cardiac Events (MCE).

Acute myocardial infarction was diagnosed according to the criteria of the European Society of Cardiology/American College of Cardiology ${ }^{21}$, considering the following two criteria sufficient for diagnosis of acute, evolving or recent myocardial infarction: (i) a typical increase and gradual decrease in cTnl concentrations or more rapid increase and decrease in creatine kinase-MB concentration in combination with at least one of the following: (a) typical ischemic symptoms, (b) development of pathological $Q$ waves in ECG, (c) ECG changes indicative of myocardial ischemia (ST segment elevation or depression), and (d) coronary artery intervention; and (ii) pathological findings of an acute myocardial infarction.

Immediately after arriving postoperatively in the ICU and every day during their stay in PACU, samples for creatine kinase-MB (CK-MB) and cTnl were collected from all patients. Samples were collected and twelve-lead ECG was performed whenever symptoms of myocardial events occurred.

All patients had continuous acquisition of leads II and V ECG to monitor and diagnose possible arrhythmias.

We also recorded PACU and hospital LOS. For mortality, we recorded PACU mortality, hospital mortality and mortality at six months after PACU discharge.

Functional capacity before surgery was evaluated in terms of the patient's ability to handle personal and instrumental ADL within the first $24 \mathrm{~h}$ after PACU admission. All eligible consenting patients were interviewed directly by a trained investigator. When the patient was unable to respond, the questionnaire was completed by a close family member living in the same household as the patient.

Health Related Quality Of Life (HRQOL) was assessed by the SF-36 ${ }^{12}$. The survey contains 36 questions that evaluate eight health domains considered to be important for patient well-being and health status. These domains reflect physical health, mental health, and the impact of health on daily functioning. The eight multiple-item domains encompass physical functioning (ten items), social functioning (two items), role limitations caused by physical problems (four items), role limitations caused by emotional problems (three items), mental health (five items), energy and vitality (four items), pain (two items) and general perception of health (five items). There is one further unscaled item relating to self-reported changes in the respondent's health status during the past year. For each item, scores are coded, summed and transformed to a scale from 0 (worst possible health state measured by the questionnaire) to 100 (best possible health state). Scores can be aggregated to measures representing a physical health summary scale (consisting of physical functioning, physical role, pain 
and general health) and a mental health summary scale (vitality, social functioning, emotional role and mental health) ${ }^{6}$.

The answers to the question about self-reported changes in health status ("compared to one year ago, how would you rate your health in general now?") were categorized as: better, about the same or worse than one year ago.

To minimize distress to the next of kin, each patient's records were checked on the hospital information system after six months to ascertain whether he or she was still alive. A copy of a formal letter was sent to all known survivors accompanied by a return envelope and a validated Portuguese SF-36 self-report form ${ }^{13,14}$. This version had been validated for the population of the city of Porto from which the subjects of this report were drawn ${ }^{22}$. Scores for all domains obtained for patients who developed MCE were compared with the published urban population values for Porto ${ }^{17}$.

We also compared the scores in all SF-36 domains obtained for MCE patients with those obtained for PACU patients who did not develop MCE after surgery.

The questionnaire used to assess dependency was based on the Katz Index of Independence in ADL 22 and Lawton Instrumental ADL scale. The Lawton Instrumental ADL scale is an easily administered assessment instrument that provides self-reported information about the functional skills necessary for living in the community. Deficits in the instrumental Lawton scale were scored and a summary score ranging from 0 (low function, dependent) to 7 (high function, independent) was obtained. The Katz ADL scale assesses basic personal activities of daily living and ranks adequacy of performance in six functions. Dependency in each personal activity was evaluated and a summary score ranging from 0 (independence in all activities) to 6 (dependency in all activities) was obtained. The personal ADL (P-ADL) considered were bathing, dressing, going to the toilet, transferring from bed to chair, continence and feeding. The instrumental ADL (I-ADL) considered were cleaning, food shopping, public transportation and cooking. Answers were categorized into two groups: able or unable to perform each activity and group of activities. Patients were considered dependent if they were dependent in at least one I-ADL or P-ADL.

The outcome endpoints considered were: (1) Functional capacity and ADL. Patients were considered dependent if they were dependent in at least one I-ADL or P-ADL. (2) Quality of life. Quality of life was evaluated at six months after PACU discharge. (3) Mortality. Patients were considered survivors if they were alive six months after PACU discharge.

Descriptive analyses of variables were used to summarize data and the Mann-Whitney $U$ test was used to compare continuous variables between groups of subjects; chi-square or Fischer's Exact test were used to compare proportions between two groups of subjects.

To evaluate the determinants of postoperative MCE and to identify independent predictors of hospital mortality, univariate analyses was performed using simple binary logistic regression with an odds ratio (OR) and its 95\% Confidence Interval $(\mathrm{Cl})$ with the following independent variables: age, gender, BMI, ASA-PS, type of surgery, comorbidities, RCRI score, type of anesthesia, intraoperative fluid administration, blood troponin
I levels at admission, length of anesthesia and temperature at admission (and MCE for mortality model). To prevent the inflation of type I error we controlled the significance level for multiple comparisons by applying Bonferroni's correction (test-wise significance level divided by the number of tests performed). All variables were deemed significant if $p \leq 0.001$.

Multiple regression binary logistics with forward conditional elimination were used to examine covariate effects for each factor in order to identify independent predictors of hospital mortality. In these models, covariates with $p \leq 0.001$ in the respective univariate analysis were entered (applying Bonferroni's correction for multiple comparisons).

A $t$ test for independent groups was used to compare population means. The SF-36 scores for the MCE population were compared with the urban population of Porto using a $t$ test.

SPSS for Windows version 16.0 (SPSS, Chicago, IL) was used to analyze the data.

\section{RESULTS}

There were 1,597 adult PACU admissions during the study period and 1,280 patients met the inclusion criteria and were followed for the development of MCE after admission. One hundred and ninety-six patients were excluded because they stayed less than $12 \mathrm{~h}$ and were not admitted overnight, 52 because they were less than 18 years old, and 44 because they were admitted more than once to the PACU; 13 were admitted after pulmonary surgery and 12 were not surgical patients.

Twenty-six patients (2\%) developed MCE during their PACU stay. The MCE cases comprised 19 acute myocardial infarctions, five cardiac arrests/ventricular fibrillation and two pulmonary edemas.

The characteristics of patients admitted to the PACU, and a comparison between the patients who did and did not deveIop MCE, are summarized in Table I. Patients who developed MCE were older (median age 72 versus 65 years, $p<0.025$ ), more likely to be ASA-PS IV/V (27\% versus $8 \%, p=0.004)$ and had RCRI scores $>2(73 \%$ versus $30 \%, p<0.001)$, had higher troponin I levels at admission $(0.59 \pm 1.36$ versus 0.05 $\pm 0.10, p<0.001)$, more frequently had hypertension $(89 \%$ versus $48 \%, p<0.001)$ and hyperlipidemia $(50 \%$ versus $28 \%$, $p=0.018)$, were more likely to have been subjected to highrisk surgery $(73 \%$ versus $46 \%, p=0.005)$, more frequently had ischemic heart disease $(69 \%$ versus $24 \%, p<0.001)$ and congestive heart disease $(77 \%$ versus $20 \%, p<0.001)$ and had received higher volumes of fluids intraoperatively $(2.0 \pm$ 3.2 versus $0.7 \pm 1.6, p<0.001$, for erythrocyte packed units, and $0.8 \pm 2.1$ versus $0.3 \pm 1.1, p=0.016$ for units of fresh frozen plasma).

Table II presents data on the severity of disease scores and outcomes. Patients who developed MCE were more severely ill (median SAPS II 30 versus 18, p $<0.001$ and median APACHE II 14 versus $8, p<0.001$ ) and stayed longer in the PACU (median LOS $84 \mathrm{~h}$ versus $20 \mathrm{~h}, \mathrm{p}<0.001$ ). The unadjusted mortality rate at six months follow-up for patients who developed MCE was nearly three times greater than for patients 
Table I - Patient Characteristics and Outcomes

\begin{tabular}{|c|c|c|c|c|}
\hline Variable & All patients $(n=1,280)$ & $\operatorname{MCE}(n=6)$ & No MCE $(1,254)$ & $p$ value \\
\hline Age in years * & $65(54-74)$ & $72(57-78)$ & $65(54-74)$ & $0.025^{\mathrm{b}}$ \\
\hline Age group ** & & & & $0.018^{a}$ \\
\hline$\geq 65$ years & $655(51)$ & $19(73)$ & $636(51)$ & \\
\hline Sex ** & & & & $0.263^{a}$ \\
\hline Male & $834(65)$ & $19(73)$ & $815(65)$ & \\
\hline Female & $446(35)$ & $7(27)$ & 439 (35) & \\
\hline ASA IV / V & $106(8)$ & $7(27)$ & $99(8)$ & \\
\hline Body mass index $\left(\mathrm{kg} \cdot \mathrm{m}^{-2}\right)^{*}$ & $25(22-28)$ & $26(24-29)$ & $25(22-28)$ & $0.071^{\mathrm{b}}$ \\
\hline General / Combined anesthesia ** & $1107(87)$ & $23(89)$ & $1184(86)$ & \\
\hline Duration of anesthesia $(\mathrm{min}){ }^{*}$ & $200(150-300)$ & $240(128-360)$ & $200(150-300)$ & $0.284^{b}$ \\
\hline Temperature at admission on PACU *** & $35.22 \pm 1.31$ & $35.14 \pm 1.28$ & $35.22 \pm 1.31$ & $0.089^{b}$ \\
\hline High-risk surgery ** & $594(46)$ & $19(73)$ & $575(46)$ & $0.005^{a}$ \\
\hline Ischemic heart disease ** & $322(25)$ & $18(69)$ & $304(24)$ & $<0.001^{a}$ \\
\hline Congestive heart disease ** & $275(22)$ & $20(77)$ & $255(20)$ & $<0.001^{a}$ \\
\hline Chronic renal failure ** & $80(6)$ & $2(8)$ & $78(6)$ & $0.491^{a}$ \\
\hline Cerebrovascular disease ${ }^{* *}$ & $179(14)$ & $6(23)$ & $173(14)$ & $0.144^{\mathrm{a}}$ \\
\hline Insulin therapy for diabetes ** & $127(10)$ & $4(15)$ & $123(10)$ & $0.253^{a}$ \\
\hline $\mathrm{RCRI}$ & & & & $<0.001^{a}$ \\
\hline$\leq 2$ & $881(69)$ & $7(27)$ & $874(70)$ & \\
\hline$>2$ & 399 (31) & $19(73)$ & $380(30)$ & \\
\hline \multicolumn{5}{|l|}{ Intraoperative fluid volume } \\
\hline Crystalloids (L) \# & $2.6 \pm 1.8(1.2-3.4)$ & $3.2 \pm 2.9(1.2-3.9)$ & $2.6 \pm 1.7(1.2-3.4)$ & $0.102^{\mathrm{b}}$ \\
\hline Colloids $(\mathrm{L})$ * & $0.2 \pm 0.4(0-0.5)$ & $0.3 \pm 0.5(0-0.5)$ & $0.2 \pm 0.4(0-0.5)$ & $0.389^{b}$ \\
\hline
\end{tabular}

a Pearson $\chi^{2}$ or Fisher's exact test; b Mann-Whitney $U$ test.

* - median (P25-75); ** $-\mathrm{n}(\%) ;{ }^{* *}-$ mean \pm sd; \# - (P25 - P75) - are the 25th and 75th percentiles.

MCE - Major Cardiac Events; RCRI - revised cardiac risk index; I-ADL - instrumental activities of daily living; P-ADL - personal activities of daily living; PACU - post anesthesia care unit.

Table II - Severity of Disease Scores, PACU and Hospital Length of Stay and Mortality

\begin{tabular}{|c|c|c|c|c|}
\hline Variable & All patients $(n=1,280)$ & $\operatorname{MCE}(n=26)$ & No MCE $(1,254)$ & $p$ value ${ }^{b}$ \\
\hline SAPS II * & $18(13-27)$ & $30(23-42)$ & $18(12-27)$ & $<0.001$ \\
\hline APACHE II * & $8(5-11)$ & $14(11-15)$ & $8(5-11)$ & $<0.001$ \\
\hline PACU length of stay (hours) * & $25(21-46)$ & $80(38-170)$ & $25(21-45)$ & $<0.001$ \\
\hline Mortality in PACU ** & $33(3)$ & $3(12)$ & $30(2)$ & 0.027 \\
\hline Mortality in hospital ** & $72(6)$ & $6(23)$ & $66(5)$ & 0.002 \\
\hline Mortality at 6 months follow-up ** & $176(14)$ & $9(35)$ & $167(13)$ & 0.005 \\
\hline
\end{tabular}

* - median (P25-75); ** - , n (\%); b - Mann-Whitney U test

MCE - major cardiac events; SAPS II - Simplified Acute Physiology Score; APACHE II - Acute Physiology and Chronic Health Evaluation; PACU - Post Anesthesia Care Unit; P25 and P75 are the 25th and 75th percentiles.

without MCE (35\% versus $13 \%, p=0.005)$. The difference was even greater for hospital mortality $(23 \%$, versus $5 \%, p=$ $0.002)$ and PACU mortality (12\% versus $2 \%, p=0.027)$.

Univariate analyses for determinants of MCE and their relevant $p$-values are summarized in Table III. The following inde- pendent predictors for development of MCE in the immediate postoperative period were identified ( $p \leq 0.001)$ : ASA-PS (OR 4.30, 95\% Cl 1.76-10.47, $\mathrm{p}=0.001$ for ASA-PS IV/V patients), hypertension (OR $8.22,95 \% \mathrm{Cl} 2.46-27.53, \mathrm{p}=0.001$ ), ischemic heart disease (OR 7.03, 95\% Cl 3.03-16.33, $p<0.001$, 
congestive heart disease (OR 13.06, 95\% Cl 5.19-32.85, p < 0.001 ) and RCRI score (OR 6.24, 95\% Cl $2.60-14.97, p<$ 0.001 for $\mathrm{RCRI}>2$ ).

Univariate analysis for severity of disease, LOS and mortality are summarized in Table IV. Severity of disease scores SAPS II and APACHE II were significantly higher in patients who developed MCE (OR 1.05, 95\% Cl 1.03-1.07, p < 0.001 and OR $1.14,95 \% \mathrm{Cl} 1.08-1.21, \mathrm{p}<0.001$ respectively). The perioperative occurrence of MCE was associated with significantly greater PACU LOS (OR 1.21, 95\% Cl 1.10-1.31, p < 0.001 ), higher hospital mortality (OR $5.40,95 \% \mathrm{Cl} 2.10-13.90$, $\mathrm{p}<0.001$ and higher mortality at six months follow-up (OR $3.45,95 \%$ Cl 1.51-7.86, $p=0.003$ ).

Multiple logistic regression analyses were used to examine the covariate effects of each factor in hospital mortality (Table V). The regression model included all variables with statistical significance in the univariate analysis for determinants of hospital mortality. This analysis showed that the development of MCE after surgery was a risk factor for hospital mortality (OR $5.40, p<0.001)$. After adjustment for age, ASA-PS, temperature at admission, high-risk surgery, congestive heart failure, emergency surgery, SAPS II, APACHE II, PACU LOS and RCRI, the independent predictors of hospital mortality were ASA-PS (OR
3.94, 95\% Cl 2.03-7.66, $\mathrm{p}<0.001$, for ASA-PS IV/V patients), high-risk surgery (OR 2.31, 95\% Cl 1.22-4.39, $p=0.011$ ), RCRI (OR 3.83, 95\% Cl 2.11-6.94, p < 0.001 for RCRI > 2) and APACHE II (OR 1.22, 95\% Cl 1.16-1.29, p < 0.001).

Three patients (12\%) died during PACU stay, six (23\%) died before hospital discharge and nine (35\%) died before follow-up at six months. Of the remaining 17 patients, four (24\%) did not answer the questionnaires at six months follow-up but were known to be alive.

For the patients who developed MCE the response rate was $76 \%$ (13 out 17 survivors at six months follow-up) and there were no statistically significant differences between participants and non-participants regarding any of the variables studied. For patients who did not develop MCE the response rate was 68\% (737 out of 1078 survivors at six months follow-up).

\section{Functional Capacity and ADL}

Six months after discharge from PACU, $69 \%$ of the patients were dependent in at least one activity in instrumental ADL and $15 \%$ in at least one personal ADL (Table VI). There was no difference in the Katz scale after surgery but dependency

Table III - Univariate Analysis for Determinants of Major Cardiac Events

\begin{tabular}{|c|c|c|c|c|}
\hline Variable & MCE $(n=26)$ & non MCE $(n=1,254)$ & Odds ratio $(95 \% \mathrm{Cl})$ & $p$ value \\
\hline Age * & $68.5 \pm 12.1$ & $62.3 \pm 15.3$ & $1.00(1.00-1.06)$ & 0.040 \\
\hline$<65$ years & $7(27)$ & $618(49)$ & 1 & \\
\hline Female & $7(27)$ & $439(35)$ & 1 & \\
\hline Male & $19(73)$ & $815(65)$ & $1.46(0.61-3.51)$ & 0.394 \\
\hline Body mass index $\left(\mathrm{kg} \cdot \mathrm{m}^{-2}\right)^{*}$ & $27.1 \pm 5.3$ & $25.4 \pm 5.8$ & $1.04(0.99-1.10)$ & 0.146 \\
\hline Emergency surgery ${ }^{\star *}$ & $8(31)$ & $268(21)$ & $1.64(0.70-3.80)$ & 0.253 \\
\hline \multicolumn{5}{|l|}{ Physical status ${ }^{\star \star}$} \\
\hline ASA II / III & $19(73)$ & $1155(92)$ & 1 & \\
\hline ASA IV / V & $7(27)$ & $99(8)$ & $4.30(1.76-10.47)$ & 0.001 \\
\hline Temperature at admission* & $35.1 \pm 1.8$ & $35.2 \pm 1.3$ & $0.96(0.72-1.28)$ & 0.756 \\
\hline Troponin at admission* & $0.59 \pm 1.36$ & $0.05 \pm 0.10$ & $25.56(3.25-200.78)$ & 0.002 \\
\hline Ischemic heart disease ${ }^{\star \star}$ & $18(69)$ & $304(24)$ & $7.03(3.03-16.33)$ & $<0.001$ \\
\hline Congestive heart disease ${ }^{\star \star}$ & $20(77)$ & $255(20)$ & $13.06(5.19-32.85)$ & $<0.001$ \\
\hline Chronic renal failure ${ }^{\star \star}$ & $2(8)$ & $78(6)$ & $1.26(0.29-5.41)$ & 0.759 \\
\hline Cerebrovascular disease ${ }^{\star *}$ & $6(23)$ & $173(14)$ & $0.88(0.74-4.73)$ & 0.184 \\
\hline Insulin therapy for diabetes ${ }^{* *}$ & $4(15)$ & $123(10)$ & $1.67(0.57-4.93)$ & 0.352 \\
\hline \multicolumn{5}{|l|}{ RCRI, n (\%) } \\
\hline$\leq 2$ & $7(27)$ & $874(70)$ & 1 & \\
\hline$>2$ & $19(73)$ & $380(30)$ & $6.24(2.60-14.97)$ & $<0.001$ \\
\hline \multicolumn{5}{|l|}{ Intraoperative fluid volume* } \\
\hline Crystalloids (L) & $3.2 \pm 2.9$ & $2.6 \pm 1.7$ & $1.16(0.97-1.39)$ & 0.103 \\
\hline Colloids (L) & $0.3 \pm 0.5$ & $0.2 \pm 0.4$ & $1.59(0.68-3.75)$ & 0.286 \\
\hline Erythrocytes (units) & $2.0 \pm 3.2$ & $0.9 \pm 1.6$ & $1.23(1.08-1.39)$ & 0.002 \\
\hline
\end{tabular}

\footnotetext{
* - mean \pm sd; ** $-\mathrm{n}(\%)$
}

$\mathrm{MCE}$ - major cardiac events; RCRI - revised cardiac risk index. 
Table IV - Univariate Analysis for Severity of Disease, Length of Stay and Mortality

\begin{tabular}{|c|c|c|c|c|}
\hline Variable & $\operatorname{MCE}(n=26)$ & non MCE $(n=1,254)$ & Odds ratio $(95 \% \mathrm{Cl})$ & $\mathrm{p}$ Value \\
\hline SAPS II* & $33.6 \pm 16.2$ & $21.1 \pm 12.7$ & $1.05(1.03-1.07)$ & $<0.001$ \\
\hline APACHE II ${ }^{*}$ & $13.8 \pm 5.5$ & $8.7 \pm 5.0$ & $1.14(1.08-1.21)$ & $<0.001$ \\
\hline PACU stay (days) ${ }^{*}$ & $4.6 \pm 3.6$ & $1.8 \pm 2.1$ & $1.21(1.10-1.31)$ & $<0.001$ \\
\hline Hospital stay (days)* & $25 \pm 27$ & $24 \pm 32$ & $1.01(0.99-1.02)$ & 0.115 \\
\hline Mortality in the PACU ** & $3(12)$ & $30(2)$ & $5.32(1.52-18.70)$ & 0.009 \\
\hline Mortality in the hospital ${ }^{* *}$ & $6(23)$ & $66(5)$ & $5.40(2.10-13.90)$ & $<0.001$ \\
\hline
\end{tabular}

${ }^{*}-$ mean $\pm \mathrm{sd} ;{ }^{* *}-\mathrm{n}(\%)$

MCE, Major Cardiac Events; SAPS II, Simplified Acute Physiology Score; APACHE II, Acute Physiology and Chronic Health Evaluation; PACU, Post Anesthesia Care Unit; $\mathrm{Cl}$, Confidence Interval.

Table V - Multivariate Regression Analysis for Predictors of In-Hospital Mortality

\begin{tabular}{llll}
\hline Variable & Simple OR & $\mathrm{p}$ & Adjusted * OR (95\% Cl) \\
\hline Age & 1.04 & $<0.001$ & - \\
Physical status & & & \\
$\quad$ ASA I / II / III & 1 & $<0.001$ & $3.94(2.03-7.66)$ \\
ASA IV / V & 13.16 & $<0.001$ & \\
Temperature & 0.70 & $<0.001$ & $2.31(1.22-4.39)$ \\
High-risk surgery & 3.44 & $<0.001$ & - \\
Ischemic heart disease & 2.24 & 0.001 & - \\
Congestive heart disease & 5.86 & $<0.001$ & - \\
Emergency surgery & 4.29 & $<0.001$ & - \\
MCE & 5.40 & $<0.001$ & $3.83(2.11-6.94)$ \\
RCRI (> 2) & 5.19 & $<0.001$ & - \\
SAPS II & 1.09 & $<0.001$ & $1.22(1.16-1.29)$ \\
APACHE II & 1.29 & $<0.001$ & - \\
PACU LOS (days) & 1.18 & $<0.001$
\end{tabular}

a Logistic regression analysis with stepwise forward method was used with an entry criterion of $p<0.05$ and a removal criterion of $p>0.1$.

MCE - major cardiac events; PACU - post anesthesia care unit; LOS - length of stay; SAPS - Simplified Acute Physiology Score; APACHE II - Acute Physiology and Chronic Health Evaluation; RCRI - Revised Cardiac Risk Index; OR - Odds Ratio; CI, Confidence Interval.

${ }^{*}$ Adjusted for age, ASA physical status, High-risk surgery, Ischemic heart disease, Congestive heart failure, Emergency surgery, MCE, SAPS II, APACHE II and PACU LOS.

Table VI - Dependency and Self-Reported Changes in Health in General, Six Months after Post Anesthesia Care Unit Discharge $(n=13)$

\begin{tabular}{llll}
\hline Activities of daily living & Before surgery & 6 months after PACU discharge & p value \\
\hline Personal activities of daily living & & $0.54 \pm 1.66$ & 0.266 \\
Katz scale * & $0.0 \pm 0.0$ & $2(15)$ & 0.018 \\
Dependency in P-ADL ** & $0(0)$ & $5.0 \pm 2.9$ & 0.003 \\
$\begin{array}{l}\text { Instrumental activities of daily living } \\
\text { Lawton scale * }\end{array}$ & $7.0 \pm 1.7$ & $9(69)$ & 0.294 \\
Dependency in I-ADL ** & $3(23)$ & \\
\hline
\end{tabular}

${ }^{*}-$ mean \pm sd; ${ }^{* *}-\mathrm{n}(\%)$.

in at least one P-ADL was higher six months after PACU discharge. The Lawton scale score was significantly lower after surgery, indicating greater dependency, but the percentages of dependency in at least one I-ADL were not different.

\section{Quality of Life Measures}

Overall, $31 \%$ stated that their level of health in general was better on the day they completed the SF-36 questionnaire than 12 months earlier, while $31 \%$ considered it to be worse (six months before PACU discharge).

Compared to values observed for the urban population of Porto, the SF-36 sub-scores of all patients who developed MCE were worse in all domains except bodily pain and mental health (Table VII).

Patients who developed MCE did not differ in any of the SF-36 domains from the other PACU patients who did not have MCE (Table VIII). 
Table VII - F-36 after Major Cardiac Event and in a General Population

\begin{tabular}{llll}
\hline SF-36 domains & 6 months after MCE & General population & $p$ value \\
\hline Physical function & $61.5 \pm 33.0$ & $75.4 \pm 23.6$ & 0.035 \\
Role physical & $51.4 \pm 32.5$ & $76.7 \pm 26.1$ & $<0.001$ \\
Bodily pain & $52.3 \pm 29.7$ & $65.7 \pm 26.2$ & 0.067 \\
General health perception & $38.8 \pm 18.3$ & $59.5 \pm 19.8$ & $<0.001$ \\
Vitality & $32.7 \pm 17.1$ & $57.2 \pm 21.1$ & $<0.001$ \\
Social functioning & $60.6 \pm 27.4$ & $76.0 \pm 24.1$ & 0.021 \\
Role emotional & $48.7 \pm 32.6$ & $76.9 \pm 25.8$ & $<0.001$ \\
Mental health & $61.2 \pm 41.6$ & $66.1 \pm 22.8$ & 0.445 \\
\hline
\end{tabular}

Values expressed as Mean \pm Standard deviation; ${ }^{a}-t$ test

SF-36, Short-form 36.

Table VIII - SF-36 after Major Cardiac Event and in a Surgical Population

\begin{tabular}{llll}
\hline SF-36 domains & 6 months after MCE $(n=13)$ & PACU surgical patients $(n=737)$ & $p$ value \\
\hline Physical function & $61.5 \pm 33.0$ & $53.4 \pm 31.3$ & 0.352 \\
Role physical & $51.4 \pm 32.5$ & $46.2 \pm 34.3$ & 0.583 \\
Bodily pain & $52.3 \pm 29.7$ & $57.0 \pm 29.8$ & 0.580 \\
General health perception & $38.8 \pm 18.3$ & $46.4 \pm 27.6$ & 0.319 \\
Vitality & $32.7 \pm 17.1$ & $34.5 \pm 16.6$ & 0.705 \\
Social functioning & $60.6 \pm 27.4$ & $57.4 \pm 30.6$ & 0.709 \\
Role emotional & $48.7 \pm 32.6$ & $50.4 \pm 34.8$ & 0.867 \\
Mental health & $61.2 \pm 41.6$ & $50.1 \pm 22.1$ & 0.081 \\
\hline
\end{tabular}

Values expressed as Mean \pm Standard deviation; ${ }^{a}-t$ test

SF-36, Short-form 36; PACU - Post Anesthesia Care Unit.

\section{DISCUSSION}

This study evaluated the occurrence of major cardiac complications after all types and magnitudes of noncardiac surgery, enrolling patients with all types of clinical risk.

The differential risk associated with various surgical procedures has been well recognized and different operations are associated with different cardiac risks ${ }^{23}$. We excluded patients submitted to cardiac or pulmonary surgery because the predicted incidence of MCE for these patients is higher ${ }^{24}$ and because at our hospital they are admitted to other ICU.

The low incidence (2\%) of serious postoperative cardiac events in our study was similar to that observed by Mangano $(3 \%)^{25}$ and Lee $(2 \%)^{5}$.

Postoperative MCE remains a leading cause of morbidity, mortality, prolonged hospital stay and increased hospital cost. In this study, we attempted to correlate and predict factors (preoperative, intraoperative and early postoperative) predisposing to MCE. The findings may enable us to predict those at high risk, so interventional measures might be planned in advance to improve outcome following such a complication, achieving a better outcome and a more efficient use of hospital and intensive care resources.

We have measured postoperative Troponin I levels for every patient although the role of the troponins for the evaluation of patients after non cardiac surgery remains unclear. Measurement of troponin I facilitates the recognition of myocardial damage but the Guidelines of the American College of Cardiology and American Heart Association for evaluating cardiac risk for noncardiac surgery do not recommend their routine measurement ${ }^{26}$.

As determinants for the occurrence of MCE this study identified five independent risk factors: ASA-PS, hypertension, ischemic heart disease, congestive heart failure and RCRI.

The ASA-PS score, a preoperative evaluation used routinely for every patient, was never intended as a perioperative risk score, but all large-scale studies have suggested that a high ASA-PS score is one of the best predictors of postoperative morbidity ${ }^{27,28}$. This indicator depends on a more generic classification of diseases that may be present, and in itself indicates the presence of comorbidities. ASA-PS classification is a strong predictor of the development of postoperative medical complications for patients in a perioperative setting.

The RCRI is a tool for predicting major cardiac complications after noncardiac surgery ${ }^{29,30}$. It was developed to predict cardiac risk on the basis of six independent prognostic factors: high risk surgery, ischemic heart disease, congestive heart disease, history of cerebrovascular disease, insulin therapy for diabetes and preoperative serum creatinine $>2.0 \mathrm{mgdL}^{-1}$. The $\mathrm{RCRI}$ score is based on predictors that are independently and significantly associated with cardiovascular events. A high score means that patients with pre-existing cardiovascular disease have an increased perioperative risk of developing MCE in postoperative period. The present study corroborated the finding by Lee et al. ${ }^{5}$ confirmed by others ${ }^{31}$ that the presence of two or more of the $\mathrm{RCRI}$ factors identified patients with increased risk for cardiac complications. In our study, RCRI was an independent predictor for development of MCE, and so were some of the factors 
included in the index such as ischemic heart disease and congestive heart failure. The contribution of heart failure to the RCRI 5 is consistent with previous studies that showed heart failure to be an important risk factor for postoperative cardiac complications ${ }^{32}$. Heart failure has been identified in several studies as associated with a poorer outcome when noncardiac surgery is performed and it is associated with a substantially increased risk during noncardiac surgery ${ }^{33}$.

Although hypertension is common, and treatment has been shown to be associated with decreased rates of death due to stroke and congestive heart disease, the Guidelines of the American College of Cardiology and American Heart Association for evaluating cardiac risk for noncardiac surgery ${ }^{23}$ considered hypertension a minor predictor, a recognized marker for cardiovascular disease that has not been proven to increase perioperative risk independently. In our study, however, hypertension appears as an independent risk factor for MCE, conflicting with numerous studies that have shown hypertension not to be independent risk factor for perioperative cardiovascular complications ${ }^{33-35}$.

In the population studied, the occurrence of an MCE was considered a risk factor for higher mortality and longer PACU stay, and therefore worse outcome. This reinforces the importance of identifying myocardial injury because it can be associated with subsequent cardiac events that carry a significant burden to the patient ${ }^{36}$. Similarly, Venkataraman et al. ${ }^{37}$ recently showed that perioperative cardiac events contributed significantly to morbidity and mortality.

In this study, patients with postoperative MCE had significantly higher hospital mortality than patients without MCE. Patients with MCE had a hospital mortality of $23 \%$, nearly five times the mortality rate of those without.

We examined the impact of the occurrence of MCE on quality of life and independence in activities of daily living. To study the impact on quality of life we used the self-evaluated health transition item in the SF-36 questionnaire. This item is not used in scoring the scales but has been shown to be useful for estimating average change in health status during the year prior to administration of the questionnaire ${ }^{38}$. Only $31 \%$ of patients with postoperative MCE stated that their quality of life was better than before surgery, indicating that only a few of the patients who developed MCE had an improved subjective perception of quality of life after surgery.
Comparisons with a general population (taken as "control") are difficult to interpret because this population was not adjusted for age or comorbidities and because surgical patients who develop MCE may view themselves as chronically ill. Thus, our finding that quality of life was worse in almost all domains in MCE patients than in the general population was not totally unexpected. PACU patients from the same urban area with similar demographic characteristics seemed more appropriate for comparison with MCE patients; for this comparison we found no differences between the two populations in any of the SF-36 domains.

The patients in our study showed higher degrees of dependency in instrumental and personal ADL after surgery. These results may be influenced by the presence of comorbidities and concurrent diseases, and as implied by the higher SAPS II and longer PACU LOS among the MCE patients this could reflect the burden still imposed by the acute changes in these patients even six months after PACU discharge.

This study has several limitations. We did not apply the SF-36 questionnaire before surgery so it was not possible to compare the quality of life of patients before and after surgery as in the study by Lloyd ${ }^{39}$. Nevertheless, we used the SF-36 question about self-reported changes in health status ("compared to one year ago, how would you rate your health in general now?") to infer that the level of health in general was better for most patients on the day they completed the SF-36 than before surgery.

In summary, this study supports the conclusion that ASAPS, hypertension, ischemic heart disease, congestive heart failure and RCRI are determinants of MCE after major noncardiac surgery. The occurrence of MCE after surgery is a risk factor for mortality and patients who developed MCE had higher mortality rates and higher PACU LOS.

Only $31 \%$ of patients who survived after surgery showed a subjective positive perception of HRQOL six months after discharge from the PACU.

Patients who developed MCE did not differ in any SF-36 domain from PACU patients who did not.

Six months after PACU discharge, a substantial number of patients were dependent in at least one activity in personal or instrumental ADL. This may be seen as an indicator of prolonged convalescence in this group of patients. 